\section{Representación del autocuidado de la salud en el trabajo de conductores de autobús urbano de Guadalajara, México}

\author{
Representation of self-care of health at work \\ among urban bus drivers in Guadalajara, Mexico
}

\section{Representação do autocuidado da saúde no trabalho de motoristas de ônibus urbano em Guadalajara, México}

\section{Resumen}

El objetivo del estudio fue analizar las representaciones sociales del autocuidado de la salud en el trabajo en conductores de autobús urbano de Guadalajara, México. La metodología fue cualitativa, con un diseño multimétodo, basado en la teoría de las representaciones sociales. En una primera etapa se efectuaron sesiones de observación participante, la información se registró en notas de campo; en una segunda fase se aplicaron las técnicas de la carta asociativa $y$ los tris jerarquizados sucesivos a 20 conductores, los datos se sometieron a análisis clásico de las asociaciones libres; en una tercera etapa se realizaron entrevistas semi-estructuradas a 4 conductores, siendo sometidas a análisis de contenido. Los resultados indican que, estructuralmente, la representación se centra en la alimentación, presentándose en niveles periféricos el descanso y el cuidado correctivo de la salud. Procesualmente, la representación gira en torno a los factores psicosociales que se desprenden de las condiciones de trabajo, el resto de las características del ejercicio laboral se consideran poco riesgosas para la salud. Las acciones de autocuidado mencionadas se enfocan en las condiciones de trabajo percibidas como riesgosas. La representación social del autocuidado de la salud en el trabajo es una construcción mental colectiva, desarrollada y modificada a través de la interacción social, la cual influye en las acciones de autocuidado empleadas frente a los riesgos existentes en el entorno laboral.

Transportes; Salud Laboral; Autocuidado

\author{
Mercedes Gabriela Orozco-Solis 1 \\ Cecilia Colunga-Rodríguez 1,2 \\ María de Lourdes Preciado-Serrano 1 \\ Mario Ángel-González 1 \\ Julio César Vázquez-Colunga 1 \\ Bertha Alicia Colunga-Rodríguez 1
}

doi: 10.1590/0102-311X00139815

\author{
Correspondencia \\ M. G. Orozco-Solis \\ Universidad de Guadalajara. \\ Juan Díaz Covarrubias 5928-204, Zapopan, \\ Jalisco 45016, México. \\ gaby_orozco_13@hotmail.com \\ 1 Universidad de Guadalajara, Guadalajara, México. \\ 2 Centro Médico Nacional de Occidente, Instituto Mexicano del \\ Seguro Social, Guadalajara, México.
}


En los entornos laborales occidentales, prevalece una ideología capitalista que privilegia la producción sobre la salud del trabajador, situación que ha favorecido el incremento de enfermedades relacionadas con el trabajo 1,2 .

Existen actividades laborales que, por las demandas propias de la actividad, implican un mayor desgaste para las personas que las desempeñan, tal es el caso de los conductores de autobús urbano, trabajadores que durante su jornada de aproximadamente 16 horas, cumplen con tareas como conducir, cobrar el costo del transporte y atender las demandas de los usuarios. Estas características del proceso productivo del conductor han sido descritas y calificadas por Evans \& Carrere 3 entre las más estresantes, dado que promueven la generación de condiciones de trabajo adversas, que propician la aparición de factores de riesgo físicos, químicos, ergonómicos y psicosociales que afectan la salud integral de este colectivo.

Gran parte de las investigaciones realizadas con esto trabajadores han partido de una postura positivista, buscando el establecimiento de relaciones entre factores de riesgo y daños a la salud $4,5,6,7,8,9,10$. Físicamente, la exposición a los factores de riesgo mencionados se relaciona con una prevalencia elevada de enfermedades respiratorias, osteomusculares, estomacales, circulatorias y crónico degenerativas como diabetes e hipertensión arterial, entre otras 4,5,11,12,13,14. Desde el punto de vista psicológico, el estrés crónico se observa con frecuencia, favoreciendo la aparición de depresión, ansiedad y síndrome de Burnout 6,15.

Los datos sugieren un impacto negativo sobre la salud, derivado de las condiciones de trabajo en las que se desempeñan los conductores, enfatizando la necesidad de desarrollar estrategias de intervención más efectivas que permitan, no sólo prevenir enfermedades, sino promocionar la salud de los trabajadores. Es así que una visión exclusivamente epidemiológica no es suficiente para conseguir este objetivo, siendo necesario profundizar em los aspectos subjetivos que influyen en las acciones de promoción de la salud 1,16,17. A pesar de la importancia que tiene la subjetividad, son escasos los estudios efectuados con conductores desde una perspectiva cualitativa, la mayor parte de los cuales ha buscado conocer la percepción que tienen acerca de sus condiciones de trabajo y de la organización en la que laboran, encontrando que existe una percepción negativa del ejercicio laboral entre los conductores, quienes perciben las condiciones de trabajo como una fuente de riesgos para su salud 8,18,19.

Este desconocimiento de la subjetividad soslaya conceptos clave para la promoción de la salud en el trabajo. Uno de ellos es el constructo de autocuidado de la salud en el trabajo, entendido como el conjunto de creencias y acciones realizadas voluntariamente por las personas con el propósito de cuidar su salud dentro del ambiente laboral 20,21. Estas prácticas de autocuidado se ven influenciadas por el bagaje cultural de las personas, quienes en vida cotidiana, a través de la interacción social, adquieren conocimientos de sentido común que orientan las decisiones que han de tomar en materia de atención y protección de su salud 21.

En el caso particular de los conductores, factores como el estrato socioeconómico y el género constituyen elementos que influyen en su concepción del autocuidado de la salud en el trabajo. Con respecto a la situación socioeconómica, es importante considerar que la mayor parte de estos trabajadores son adultos que pertenecen a un estrato bajo, quienes suelen tener la creencia de que el cuerpo es una herramienta de trabajo usada para la obtención de un ingreso monetario 22 . El género culturalmente constituye otro factor relevante en diversas sociedades, donde se asignan al hombre atributos de masculinidad, como fortaleza física, donde el deseo de autocuidarse puede asociarse a condiciones de debilidad que son percibidas como poco varoniles 23 .

Tomando em cuenta que el autocuidado de la salud en el trabajo es un constructo subjetivo, derivado de la interacción social, consideramos que las representaciones sociales son un medio para acercarse a comprender las decisiones de las personas, siendo entendidas como el resultado del proceso por medio del cual se construyen y organizan mentalmente los conocimientos, adquiridos a través de la interacción social con los grupos con quienes el individuo convive cotidianamente, el cual influye de manera directa en sus comportamientos 24,25 .

La principal aportación de este tipo de estudios es el poder identificar las discrepancias entre las representaciones del autocuidado de la salud en el trabajo que tienen los profesionales de la salud y los propios trabajadores. El estudio de las representaciones sociales en el campo de la salud permite acceder a las maneras de pensar y de actuar de las personas en lo cotidiano, conocimientos que podrían llegar a ser muy útiles para el desarrollo de estrategias de prevención que permitan alcanzar 
una mayor promoción de la salud, menor gasto de recursos y, sobre todo, una mejor calidad de vida para los trabajadores 26,27 .

Por lo tanto, el propósito de este estudio fue analizar las representaciones sociales del autocuidado de la salud en el trabajo en conductores de autobús urbano de Guadalajara, Jalisco, México.

\section{El contexto}

La investigación se llevó a cabo en la ciudad de Guadalajara, considerada como la segunda ciudad más grande del país, con una población aproximada de 1.495.189 habitantes 28. Los principales medios de transporte públicos dentro de la ciudad son los autobuses urbanos y el sistema de tren eléctrico urbano 29 , siendo el primero el que mantiene una mayor demanda, debido a la amplitud del área de servicio.

Dentro de la ciudad circulan aproximadamente cinco mil unidades de transporte urbano 30 , cada una de las cuales es operada por dos trabajadores, predominantemente varones. Las unidades se encuentran organizadas dentro de un promedio de 218 rutas de autobús, pertenecientes a alguna de las 17 empresas que operan este medio de transporte ${ }^{31}$. Del total de las rutas de la ciudad, se seleccionaron tres para participar en la investigación, las cuales convergen en los mismos escenarios para los trabajadores que laboran em ellas: la unidad vehicular, la base y la terminal correspondientes a la ruta.

La unidad vehicular es el lugar donde el trabajador realiza sus actividades principales que involucran el conducir y atender al pasajero. La base es el espacio donde los conductores que trabajan en estas rutas, acuden al inicio y término de su jornada laboral a entregar y recibir la unidad vehicular. La terminal es el lugar donde el trabajador llega cada vez que finaliza el circuito que comprende su ruta, una persona que labora en la terminal registra el tiempo que le tomó al conductor realizar el recorrido, si les sobran unos minutos, los conductores puede realizar actividades como comer o acudir a los servicios sanitarios, no obstante, si llegaron retrasados en comparación con el tiempo establecido, tienen que salir inmediatamente a retomar el recorrido.

\section{Metodología}

La investigación fue de tipo cualitativo con niveles de análisis interpretativo, utilizando un diseño multimétodo, basado en la teoría de las representaciones sociales. El estudio tuvo una duración de 3 años, completándose en su totalidad durante el periodo 2009-2012.

El trabajo fue revisado y aprobado por el comité de ética de la Maestría en Ciencias de la Salud en el Trabajo de la Universidad de Guadalajara. Se constituyó por tres fases, de manera inicial se efectuaron sesiones de observación participante, donde se incluyeron, utilizando un muestreo por oportunidad, a los conductores que se encontraran presentes en el escenario de la investigación. La segunda fase tuvo como objetivo estudiar la representación social desde un enfoque estructural, para este propósito se incluyeron 20 individuos ${ }^{32}$, seleccionados por medio de un muestreo propositivo 33 . La etapa final utilizó un enfoque procesual en los procedimientos de recolección y análisis de la información, en ella se incluyeron 4 informantes clave, seleccionados por medio de un muestreo en cadena ${ }^{34}$. Como criterios de inclusión, se integraron los trabajadores que se encontraban laborando en el puesto de conductor de autobús por lo menos con un año de antigüedad 24,25 (Figura 1).

Adicionalmente, se realizaron nueve observaciones, tres de las cuales se llevaron a cabo al inicio de la jornada de trabajo, tres al finalizar y tres durante recorridos completos de los camiones de las rutas incluidas. Durante cada sesión, se completó un registro de observación por medio de un protocolo previamente diseñado, además, se utilizó un diario de campo para registrar información relevante a través de notas de campo (metodológicas, teóricas y anecdóticas) ${ }^{35}$. Los registros de las observaciones se transcribieron en formato electrónico para, posteriormente, ser impresos y analizados.

Una vez finalizada la fase de observación, se dió inicio a la aplicación de la técnica de la carta asociativa a los participantes 36 , quienes previamente recibieron información sobre los propósitos de la investigación, obteniendo un consentimiento informado verbal para participar en el estudio, indicando que la participación era voluntaria y confidencial. 


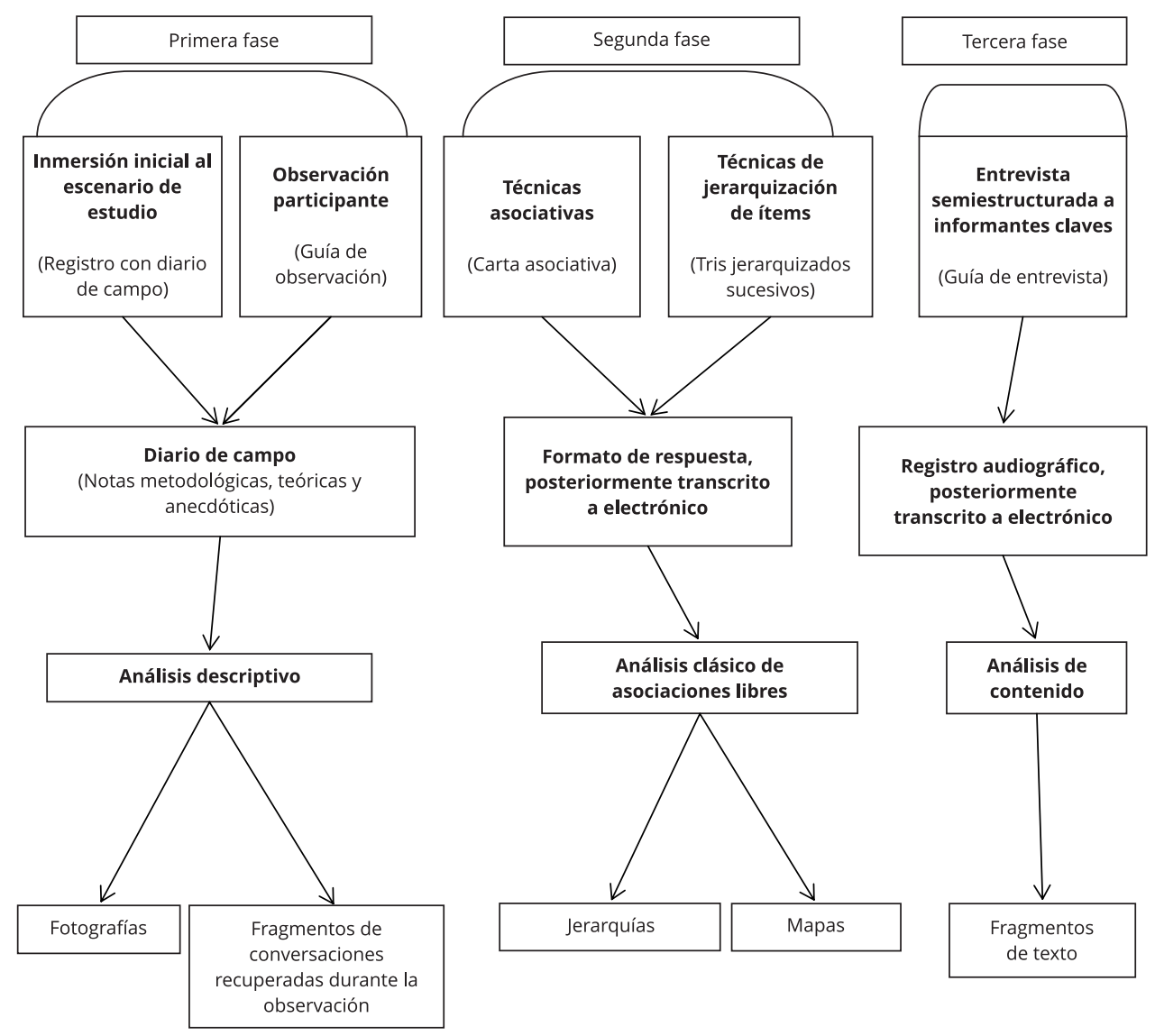

La carta asociativa consta de tres etapas:

1. En un primer momento, a partir de un término inductor, diseñado con anterioridad, se le pide a la persona que realice todas las asociaciones libres que le vengan a la mente.

2. Después de esta recolección de asociaciones, se le pidió a la persona que generara una segunda serie, solamente que esta vez se partió de un par de palabras que contuvieran, por una parte, el término inductor inicial y cada uno de los elementos asociados, producidos por el participante en la primera fase.

3. Cada una de las cadenas asociativas nuevas fue utilizada para solicitar nuevas asociaciones de parte de la persona 36,37 .

La información obtenida fue transcrita a formato electrónico para ser analizada a manera de asociación libre. Asimismo, se obtuvieron los índices de frecuencia, de rango y la relación existente entre ellos 36,37 .

A partir de los resultados obtenidos, se seleccionaron los 32 elementos que presentaron una mayor frecuencia de aparición, lista que fue utilizada para la aplicación de la técnica de tris jerarquizados sucesivos. De manera inicial, cada frase se escribió en una ficha. El total de las frases fueron proporcionadas a los participantes con la primera indicación de que eligieran los 16 elementos más característicos del objeto estudiado (autocuidado de la salud en el trabajo) y los 16 menos relacionados con el 
tema. A partir de los 16 ítems seleccionados como más característicos, se repitió la operación, pidiéndoles que seleccionara los 8 más relacionados y los 8 menos característicos, y así sucesivamente. La acción de partir por mitad se repitió hasta que quedaron dos fichas para elegir la frase más representativa del tema y la menos relacionada, obteniendo una clasificación por orden de importancia de cada participante 36,37. Los datos obtenidos fueron registrados por escrito en su correspondiente formato, para posteriorment ser analizados por medio de un análisis clásico de las asociaciones libres 36,37.

Una vez concluida la segunda etapa, se inició la fase final, donde se aplicaron entrevistas semiestructuradas a cuatro conductores, seleccionados como informantes clave por los propios participantes de la etapa anterior. Previo al inicio de la entrevista, se le explicaron a cada participante los objetivos de la investigación y se les solicitó su consentimiento verbal, aclarando que la participación era voluntaria y confidencial. Las entrevistas fueron basadas en una guía previamente elaborada con base en los resultados obtenidos de las fases anteriores, mantuvieron una duración máxima de una hora, siendo necesaria únicamente una sesión con la mayor parte de los informantes. Los registros auditivos audiográficos provenientes de las sesiones de entrevista fueron transcritos electrónicamente. La identidad de la persona que brindó la información durante la entrevista fue manejada de manera confidencial, siendo sustituida por otro nombre clave, únicamente la persona que realizó la entrevista conoce la identidad real del entrevistado.

La información proveniente de las entrevistas fue transcrita y sometida a un análisis de contenido 38 , siguiendo el proceso de trascripción- revisión - impresión - lectura - codificación construcción de categorías- relectura tanto longitudinal como transversal - saturación- categorías de análisis -cuadros de concentración de información.

A partir de la información recabada en las entrevistas emergieron las siguientes categorías de análisis:

Autocuidado de la salud en el trabajo: información relacionada con las actividades de autocuidado de la salud en el trabajo, actitud respecto a su importancia como mecanismo de protección de la salud y valoración de las conductas de autocuidado de la salud en el trabajo empleadas cotidianamente.

Agentes de riesgo identificados en el trabajo: información mantenida respecto a los riesgos de trabajos físicos, químicos, ergonómicos y psicosociales a los que se encuentran expuestos, actitud respecto a su importancia como factores de riesgo para la salud y valoración de la importancia de efectuar estrategias de autocuidado frente a ellos.

\section{Resultados}

En la muestra final se incluyeron 24 participantes, en su totalidad varones, con una edad promedio de 43 años y una escolaridad predominante de secundaria; laboralmente mantuvieron una antigüedad media de 15 años desempeñando este ejercicio laboral y 10 años realizando el trabajo en la ruta en la que laboran actualmente.

\section{El contexto del conductor de autobús urbano}

A partir de la observación participante se identificó que, dentro de los espacios en los que el conductor realiza su actividad laboral se cuenta con pocas oportunidades para ejercer acciones de autocuidado, debido principalmente a que las condiciones de trabajo no lo permiten. Por ejemplo, se observó que los tiempos para consumir alimentos no se encuentran establecidos dentro del horario de trabajo, el conductor puede comer solamente si le sobra tiempo entre sus recorridos, por lo cual suelen consumir comida de fácil acceso, alta en grasa, café y carbohidratos; asimismo, los servicios sanitarios suelen estar limitados a los momentos que les quedan libres.

Dos de las condiciones de trabajo que llaman la atención son los horarios y la forma de pago. Las jornadas laborales tienen una duración aproximada de 24 horas consecutivas de trabajo, seguidas por 24 horas de descanso, donde en un turno regular, el trabajador conduce un promedio de 12 horas seguidas. Respecto al salario, en las rutas participantes se cuenta con un pago fijo, sin embargo, en la mayor parte de las rutas de la ciudad los conductores reciben un pago proporcional al número de pasajeros atendidos en el día. 
Durante los recorridos realizados en las rutas participantes, se identificó la presencia de conductas de manejo que pueden resultar riesgosas, como el sobrepasar los límites de velocidad, adelantar outros vehículos abruptamente e interactuar agresivamente con conductores. Desde la opinión de los trabajadores, estos comportamientos se generan debido a la presión que les imprime dos situaciones: (i) alcanzar una venta alta de boletos; (ii) tener tiempo para comer en la tarde.

Uno de los recorridos se realizó con un trabajador de un año de antigüedad, donde se pudo vislumbrar un concepto de salud-enfermedad, basado en la suerte y en la predisposición genética, realizando comentarios que indicaban que las condiciones presentes en su ejercicio laboral no implican un factor de riesgo para su salud, y que cuando a una persona le toca enfermarse no importa las acciones que realice para prevenirlo: "Cuando te toca, te toca y no hay nada que puedas hacer para evitarlo" (25 años de edad y 1 año de antigüedad en el puesto).

Por el contrario, los conductores de mayor antigüedad consideraron que, debido a las características de su ejercicio laboral, el conducir un autobús es un trabajo desgastante, dado que existen condiciones que pueden llegar a ocasionarles un daño a su salud física y mental, tales como el no poder comer a una hora determinada o no poder tomar agua.

"Este trabajo te cambia física y mentalmente" (37 años de edad y 15 de antigüedad en el puesto).

Otro dato importante que se recopiló de la observación fue la alta presencia de enfermedades crónico-degenerativas, sobre todo de diabetes e hipertensión.

\section{La alimentación, elemento nuclear de la representación}

A partir de las técnicas estructurales que fueron utilizadas, se obtuvo un mapa que plasma la forma en que la representación del autocuidado de la salud en el trabajo se estructura para este colectivo.

De la aplicación inicial de la carta asociativa se obtuvieron 86 frases, las mismas que fueron agrupadas en 11 categorías para su posterior análisis (Tabla 1). De este listado, solamente 27 frases presentaron una frecuencia superior al 2\%. Debido a que se necesitaban 32 frases para la aplicación de la técnica de los tris jerarquizados sucesivos, en una segunda ocasión, se les proporcionó a los mismos participantes la lista de las 59 frases que obtuvieron una sola mención, pidiéndoles que eligieran las 5 que consideraran más relacionadas con el concepto de autocuidado de la salud en el trabajo. Posteriormente, se efectuó un análisis de frecuencia de los elementos seleccionados, eligiendo los cinco que mantuvieron una mayor mención para ser integrados a la lista de las frases.

Las frases que se incluyeron en la lista para completar los 32 elementos fueron:

- Buena alimentación.

- Saber dormir.

- Menos refresco.

- Baños limpios.

- Actividad física.

A continuación, se llevó a cabo la aplicación de la técnica de los tris jerarquizados sucesivos, la cual tuvo la finalidad de identificar la forma en la que se organizan dentro de la representación estudiada, los elementos encontrados por medio de la carta asociativa. En los resultados se observó que los conceptos que permanecieron hasta la última selección se encuentran relacionados con las categorías de alimentación, líquidos, descanso, salud y enfermedad (Tabla 2).

Por medio del análisis realizado, fue posible identificar que dentro de la representación social del autocuidado de la salud en el trabajo en los conductores, el elemento nuclear es la alimentación, a partir de la cual se van alineando el resto de los componentes periféricos, uno es el descanso entendido como dormir, el otro es el cuidado de la salud, comprendido desde una perspectiva correctiva que implica tomar regularmente medicamentos para control de enfermedades crónicas (Figura 2).

\section{Autocuidado de la salud en el trabajo, control de emociones}

Procesualmente, la alimentación es percibida como un aspecto importante del autocuidado, no obstante, los participantes concuerdan en que, debido a las condiciones de trabajo en las que laboran, donde carecen de un tiempo destinado para consumir alimentos durante la jornada, es común que consuman comida poco saludable, ya que, "es eso, o no comer nada”. Por lo tanto, la alimentación 


\section{Tabla 1}

Categorías de clasificación de los elementos obtenidos de la aplicación de la carta asociativa.

\begin{tabular}{lc}
\hline Categoría & Número de frases incluidas \\
\hline Alimentación & 20 \\
Descanso & 13 \\
Líquidos & 10 \\
Aseo & 7 \\
Salud sexual & 1 \\
Ejercicio & 6 \\
Estrés & 10 \\
Cuidar a otros & 3 \\
Hábitos & 4 \\
Salud & 5 \\
Enfermedad & 7 \\
\hline
\end{tabular}

Nota: número de informantes $=20$.

Fuente: directa por cuestionario.

Tabla 2

Frecuencias que se refirieron en la quinta (última) selección de las categorías.

\begin{tabular}{ll}
\hline $\begin{array}{l}\text { Categoría en que se engloba/ } \\
\text { Última selección }\end{array}$ & Frecuencia con que se repite \\
\hline Alimentación & 6 \\
$\quad$ Alimentación & 2 \\
$\quad$ Comer a tus horas & 2 \\
$\quad$ Comer frutas y verduras & \\
Descanso & 3 \\
$\quad$ Descanso & 1 \\
Dormir & \\
Salud & 3 \\
Cuidar de la salud & \\
Líquidos & 2 \\
Agua & \\
Enfermedad & 1 \\
Medicamentos & \\
\hline
\end{tabular}

Nota: número de informantes $=20$.

Fuente: directa por cuestionario.

pasa a un segundo término, encontrando una representación del autocuidado de la salud en el trabajo definida principalmente como "tratar de no enojarse", donde el controlar los estados emocionales es valorado como central en el constructo, dado que el "no saber controlar las emociones" es percibido como uno de los comportamientos más riesgosos por los conductores, puesto que desde su perspectiva, esta situación origina la aparición de alteraciones importantes en la salud.

"La alimentación, el dormir bien, el descansar, el tener un entretenimiento, ya sea con tu familia, amigos, todo eso es importante, pero jno enojarte!, jel tratar de no enojarte! Es fundamental” (29 años de edad). 


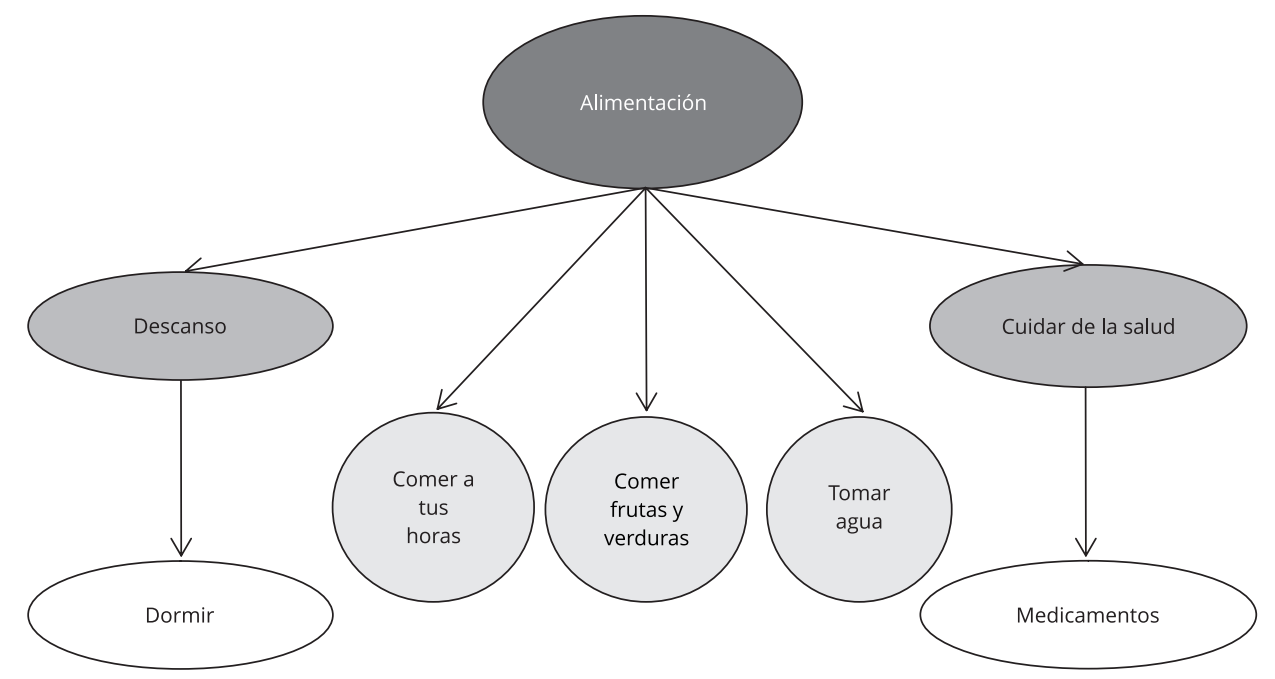

\section{Agentes de riesgo identificados en el trabajo}

En cuanto a los conocimientos, prácticas y valoración que los conductores tienen acerca de los factores de riesgo presentes en su entorno laboral, derivado de las condiciones de trabajo, se encontró que los factores psicosociales y físicos, específicamente el ruido, son percibidos como riesgosos, sin embargo, el resto de las condiciones de trabajo que pudieran representar un factor de riesgo (contaminación ambiental, postura física, etc.) no se consideran como un peligro para su salud, al ser percibidas como una parte normal del trabajo.

Destaca el hecho de que los factores de riesgo psicosociales, originados por las condiciones en las que laboran estos trabajadores, se consideran como los más riesgosos para la salud, identificando al trato con los usuarios como el elemento "más difícil" de manejar dentro de su ejercicio laboral. El verse expuestos a estas condiciones se relaciona con la aparición de enfermedades crónico-degenerativas, como la diabetes e hipertensión, favorece las agresiones entre conductores e inclusive aumenta las posibilidades de verse involucrados en accidentes automovilísticos.

"Por ejemplo, uno en la situación que tenemos de conductor de servicios públicos, pues no hay día que no nos rayen en la madre de diez a veinte o a veces hasta más veces todo el día y todos los días, y si se pone uno a escucharlos, pues hay veces que se enciende uno ooo, te estresas se te sube la sangre, también si las contestas o les das un cerrón (...)" (50 años de edad).

Con respecto al resto de las condiciones de trabajo que favorecen la generación de riesgos químicos, ergonómicos y físicos, se encontró que hay escaso consenso, con respecto a si estos son evaluados como de riesgo para la salud, ya que algunos de los participantes las identificaban como una parte natural de su trabajo, mientras otros las consideraban las principales responsables de diversas alteraciones físicas.

\section{Prácticas de autocuidado de la salud en el trabajo}

Las prácticas de autocuidado de la salud en el trabajo mencionadas por los informantes fueron variadas, incluyendo acciones como el uso de desparasitantes, vitaminas, uso de lentes obscuros para el cuidado de la vista, de bloqueador solar y evitar trabajar turnos de más. 
"En mi trabajo, la vista, (...) casi desde que inicie este trabajo de conductor, que ya son casi veinte años, de esos veinte años, tengo, pues que serán, pues yo digo que casi diecinueve o diecinueve y medio, usando lente obscuro para protegerme del sol (...) para mis brazos no uso manga larga, pero siempre me pongo bloqueador" (50 años de edad).

Debido a la importancia que tienen los aspectos psicosociales en el trabajo, al hablar del tema de autocuidado de la salud, fue posible observar acciones enfocadas especialmente a protegerse frente a factores psicosociales, haciendo referencia a emplear estrategias como consumir ciertos alimentos, ignorar a los pasajeros que los molestan y trabajar solamente en los turnos indicados.

"Ya los viejos tenemos ya remedios caseros, que si me asusté o si me enojé lo que hago es buscar una tienda en lo primero o en la calle o en donde sea un bolillo dicen que eso es muy bueno para recoger las bilis, comérmelo sin tomar agua, ni tomar mmm..., líquidos, eso es para prevenir un diabetes (...) eso es para prevenir según yo el diabetes" (60 años de edad).

Cabe señalar que en México, el bolillo es un tipo de pan elaborado con harina de trigo y levadura, puede ser salado, pero no se le considera como pan dulce. Llama la atención que a pesar de que los conductores refieren aplicar acciones de autocuidado frente a algunas condiciones de trabajo que perciben como riesgosas, la mayor parte de las alteraciones físicas y mentales que refieren como asociadas al trabajo se consideran inevitables, entendiendo el daño como una parte natural de la vida laboral a la que se tienen que acostumbrar para poder continuar trabajando.

"Pero son desgastes que, pues ahí, los vamos asimilando y acostumbrándonos y aprendiendo a vivir con ellos, pues ya qué, porque si no, qué comes" (60 años de edad).

\section{Discusión}

Toda representación social cuenta con características estructurales y de contenido que son específicas para el grupo dentro del cual se genera la representación, pues parten de la interacción social, dichos elementos algunas veces son diferentes, pues hacen referencia a aspectos cognitivos de distinto nivel.

Desde el punto de vista estructural, la alimentación aparece como elemento nuclear de la representación del autocuidado de la salud en el trabajo, mientras que en niveles periféricos se presenta el descanso y el cuidado de la salud desde un punto de vista correctivo, que implica el uso de medicamentos para el control de enfermedades crónicas. Estos resultados concuerdan con lo encontrado por diversos autores, quienes reportan que la alimentación y el descanso suelen ser valorados como importantes cuando se exploran conceptos de autocuidado con trabajadores, lo cual, pudiera deberse por un lado a la influencia que tienen los medios de comunicación, donde constantemente se refuerza la importancia de mantener una dieta saludable, y por otro, a la propia experiencia de los trabajadores, donde las personas que presentan una privación contante de sueño reparador, ocasionado por el trabajo, como por ejemplo los médicos, son las que tienden a mencionar el descanso como importante $21,39,40$.

En cuanto al contenido de la representación del autocuidado de la salud en el trabajo, desde una perspectiva procesual, se observó que el control de estados emocionales, específicamente el estrés y el enojo, aparecen como aspectos importantes dentro de esta representación. La característica laboral que se consideró como riesgosa, desde el punto de vista psicosocial, es el contacto directo con los pasajeros. $\mathrm{Al}$ respecto encontramos que en estudios realizados con profesionales que se enfrentan a condiciones que implican la interacción con otras personas, como los docentes y médicos, los participantes suelen darle una mayor importancia a los aspectos psicosociales cuando se habla acerca del autocuidado de la salud, rescatando la necesidad de cuidar su salud mental frente a las condiciones de trabajo 41,42. Para los conductores, el controlar el estrés ocasionado por la interacción con los usuarios y otros conductores de vehículos es concebido como un aspecto trascendental del autocuidado, debido a que, según la creencia popular, el enojarse favorece la aparición de alteraciones a la salud, que van desde migrañas hasta enfermedades crónico-degenerativas. Este resultado coincide con lo reportado por Torres 26 , quien señala que en el saber popular la diabetes es atribuida, principalmente, a factores de orden emocional como los sustos y los corajes.

Dentro de las entrevistas se abordó también la representación que los conductores tienen acerca de las características de su trabajo, de manera que fuera posible identificar las condiciones que 
consideran riesgosas y en las que refieren emplear acciones de autocuidado. Entre los resultados se observó que, a excepción de las condiciones de trabajo que generan factores de riesgo psicosociales y físicos, en específico, el ruido, el resto de las características del entorno laboral son consideradas como poco riesgosas. Inclusive, en los casos en que las condiciones se consideraron adversas para la salud, el daño es definido como inevitable, siendo escasas las acciones de autocuidado que refieren emplear frente a ellas. Este aspecto podría ser retomado dentro de los programas de prevención destinados a estos trabajadores, ya que como lo señala Ojeda 43, el autocuidado surge de un proceso de toma de conciencia personal en el cual se percibe el riesgo y se toman decisiones acerca de las acciones que han de implementarse ante tal agente, por lo tanto, la falta de la percepción del riesgo favorece la realización de la conducta peligrosa 44,45,46,47.

Las limitaciones de esta investigación son las propias de las investigaciones enfocadas al estudio de las representaciones sociales. La primera de ellas hace referencia al constante proceso de cambio en el cual se encuentran las representaciones, donde a través de la interacción, su contenido se modifica, dándole un carácter temporal al conocimiento adquirido acerca de las mismas. Para amortiguar esta limitación se realizó un análisis estructural de la representación, dentro del cual se considera que los elementos nucleares identificados forman parte del esqueleto central, siendo modificados dentro de un proceso más lento que los encontrados en niveles periféricos, proporcionándole una mayor consistencia a los resultados obtenidos en esta investigación. Asimismo, otra de las limitaciones encontradas es que la representación social suele servir solamente como guía de acción para el comportamiento, por lo cual es imposible distinguir la forma en que el contenido encontrado en la representación es plasmado en las prácticas, algunas de las cuales se refirieron entre los comentarios expresados por los participantes 48 . Debido a esta situación, el análisis de la representación se acompañó de observación participante, de tal forma que fuera posible identificar previo a la fase de entrevista, prácticas que pudieron mencionarse en la interacción con los informantes.

A partir de los resultados presentados, es posible abrir nuevos cuestionamientos respecto a las prácticas que realmente realizan estos trabajadores para cuidar su salud dentro del entorno laboral y los cambios que pudiera haber en sus representaciones, producidos por el acceso a nueva información o interacciones sociales. Asimismo, se rescata la importancia de conocer la representación del autocuidado de la salud en el trabajo de los dueños de las concesiones, de manera que sea posible entender la visión existente en los dos principales actores del proceso y, a partir de ahí, poder emitir sugerencias y estrategias de mejora enfocadas a proteger la salud de este vulnerable grupo.

\section{Colaboradores}

M. G. Orozco-Solis y C. Colunga-Rodríguez participaron en la elaboración y revisión del artículo. M. L. Preciado-Serrano y M. Ángel-González colaboraron en la revisión de redacción de artículo. J. C. Vázquez-Colunga y B. A. Colunga-Rodríguez apoyaron en la revisión final del artículo.

\section{Agradecimientos}

Un agradecimiento a la Universidad de Guadalajara y al Consejo Nacional de Ciencia y Tecnología, así como a aquellas personas que participaron en este estudio compartiendo con nosotros su tiempo, conocimiento y experiencias. 


\section{Referencias}

1. Muñoz A, Castro E. De la promoción de la salud a los ambientes de trabajo saludables. Salud Trab (Maracay) 2010; 18:141-52.

2. Secretaría de Trabajo y Previsión Social. Información sobre accidentes y enfermedades de trabajo nacional 2005-2014. http://autogestion. stps.gob.mx:8162/pdf/Nacional\%202005-2014. pdf (accedido el 24/Feb/2016).

3. Evans G, Carrere S. Traffic congestion, perceived control, and psychophysiological stress among urban bus drivers. J Appl Psychol 1991; 9:27-46.

4. Tovalin H, Lazcano F. Las condiciones de salud de los conductores de autotransportes urbanos de pasajeros de la ciudad de México. Bol Oficina Sanit Panam 1991; 111:324-32.

5. Chaparro P, Guerrero J. Condiciones de trabajo y salud de una empresa de transporte público urbano en Bogotá D.C. Rev Salud Pública 2001; 3:171-87.

6. Carbonell E, Bañuls R, Miguel J. El ambiente de tráfico como generador de ansiedad en el conductor: Inventario de Situaciones Ansiógenas en el Tráfico (ISAT). Anu Psicol 1995; 65: 165-83.

7. Ferreira V, Martin E, Guimarães M, Barbosa L. Lumbalgia y factores psicosociales en conductores de autobús urbano. Fitness \& Performance Journal 2006; 5:295-9.

8. Lima C, Juárez A. Un estudio exploratorio sobre estresores laborales en conductores de transporte público colectivo en el Estado de Morelos, México. Cienc Trab 2008; 30:126-31.

9. Gutiérrez J, Sierra J, Alfaro O. Agresividad al volante en el transporte público de San Salvador: estudio de factores asociados. San Salvador: Universidad Tecnológica de El Salvador; 2008.

10. Alperovitch-Najenson D, Katz-Leurer M, Santo Y, Golman D, Kalichman L. Upper body quadrant pain in bus drivers. Arch Environ Health 2010; 65:218-23.

11. Rayo V. La salud laboral en conductores de autobús urbano, una tarea pendiente. Barcelona: Centre d'Ergonomia i Prevenció, Universitat Politècnica de Catalunya (UPC); 2013.

12. Razmpa E, Khosro S, Babak S. Urban bus drivers' sleep problems and crash accidents. Indian J Otolaryngol Head Neck Surg 2011; 63:269-73.
13. Saberi H, Moravveji A, Fakharian E, Kashani M, Dehdashti A. Prevalence of metabolic syndrome in bus and truck drivers in Kashan, Iran. Diabetol Metab Syndr 2011; 3:8.

14. Portela B, Queiroga M, Constantini A, Zannin $\mathrm{H}$. Annoyance evaluation and the effect of noise on the health of bus drivers. Noise Health 2013; 15:301-6.

15. Salas C, Meliá J. Evaluación de riesgos psicosociales y factores psicosociales que afectan a la probabilidad de accidente en una empresa de transporte urbano de economía social. In: Tercer Congreso Internacional de Riesgos Laborales. http://www.uv.es/meliajl/ Papers/2004SalasMelia.pdf (accedido el 24/ $\mathrm{Feb} / 2016)$

16. Rodríguez J. Propuesta: Programa Integral de Autocuidado dirigido a personal de enfermería. Enferm Costa Rica 2010; 31(1). http://www.binasss.sa.cr/revistas/enfermeria/v31n1/art2.pdf.

17. Fonseca AES, Valdés YD, Del Toro CE. Intervención psicoeducativa para el autocuidado desde una perspectiva de género en el sector. In: Convención Internacional de Salud Pública. http://www.convencionsalud2015.sld. cu/index.php/convencionsalud/2015/paper/ view/314/939 (accedido el 24/Feb/2016).

18. Arango M, González C. Estudio de las representaciones sociales que tienen los conductores de bus urbano acerca de la empresa de transporte de Santa Fé, Bogotá. Bogotá: Pontificia Universidad Javeriana; 1997.

19. Rey de Castro J, Soriano S. Hipersomnia durante la conducción de vehículos ¿causa de accidentes en carreteras? A propósito de un estudio cualitativo. Rev Soc Peru Med Interna 2001; 15:49-142.

20. Orem D. Nursing: concepts of practice. 5th Ed. St. Louis: Mosby Year Book; 1995.

21. Cardona JA, Díaz ED, López YL. Autocuidado en recicladores-recuperadores informales de Medellín, Colombia, 2005. Rev Fac Nac Salud Pública 2009; 27:309-16.

22. Keijzer B. Hasta donde el cuerpo aguante: género, cuerpo y salud masculina. Revista Internacional de Estudios sobre Masculinidades 2004; I:137-52. 
23. Aguilar J. Masculinidades, choferes y espacio urbano en México. Revista de Ciencias Sociales 2003; 10:201-24.

24. Moscovici S. El psicoanálisis, su imagen y su público. Buenos Aires: Huemul; 1961.

25. Moscovici S. Des représentations collectives aux representations sociales: éléments pour une histoire. In: Jodelet D, editor. Les répresentations sociales. 7th Ed. Paris: Presses Universitaires de France; 2003. p. 79-103.

26. Torres T. Sangre y azúcar: representaciones sociales sobre la diabetes de los enfermos crónicos en un barrio de Guadalajara. Guadalajara: Universidad de Guadalajara; 2004.

27. Herrera J, Torres T. Las representaciones sociales de la salud y enfermedad en el trabajo de jornaleros agrícolas del Municipio de Aguililla, Michoacán. Revista Interamericana de Educación de Adultos 2012; 34:43-61.

28. Ramírez G. Zona Metropolitana de Guadalajara Jalisco. http://www.jalisco.gob.mx/es/jalisco/ guadalajara (accedido el 24/Feb/2016).

29. DINAMIA Investigación Social Estratégica. Transporte público en Guadalajara. http:// www.elpoderdelconsumidor.org/wp-content/ uploads/Reporte-EPC-Guadalajara-Prensa130409.pdf (accedido el 24/Feb/2016).

30. García A. Avanza 75\% la revisión a unidades de transporte público. El Informador 2010; 22 jul. http://www.informador.com.mx/jalis co/2010/211473/6/avanza-75-revision-a-uni dades-del-transporte-publico.htm (accedido el 24/Feb/2016).

31. Gobierno del Estado de Jalisco. 240 rutas del transporte público de Guadalajara. http://www. jalisco.gob.mx/es/prensa/noticias/14284 (accedido el 24/Feb/2016).

32. Weller S, Kimball A. Systematic data collection. Thousand Oaks: Sage Publications; 1988.

33. Arcury T, Quandt S. Qualitative methods in arthritis research: sampling and data analysis. Arthritis Care Res 1998; 11:66-74.

34. Hernández R, Fernández C, Baptista P. Metodología de la investigación. 4a Ed. México DF: McGraw Hill; 2007.

35. Bernard H. Researchmethods in cultural anthropology. Newbury Park: Sage Publications; 1988.
36. Araya S. Las representaciones sociales: ejes teóricos para su discusión. San José: Facultad Latinoamericana de Ciencias Sociales; 2002.

37. Abric J. Prácticas sociales y representaciones sociales. México DF: Ediciones Coyoacán; 1994.

38. Bardin L. El análisis de contenido. Madrid: Akal; 1996.

39. Bamonde M, Morales H, López G. Nivel de aplicación de las acciones de autocuidado de la salud por personal de una unidad médica. Revista de Enfermería del IMSS 2003; 11:77-81.

40. Arenas L, Hernández I, Valdez R, Bonilla P. Las instituciones de salud y el autocuidado de los médicos. Salud Pública Méx 2004; 46:326-32.

41. Lavoignet B, Torres T, Soltero R, Aguilera M. El autocuidado de la salud y sus significados sociales en docentes universitarias de México. Enfermería del Trabajo 2015; 5:44-51.

42. Cruz M, Chaves M, Marchi R, Almeida L, Oliveira I, Pedrão L. Exceso de trabajo y agravios mentales a los trabajadores de la salud. Rev Cuba Enferm 2010; 26:52-64.

43. Ojeda T. EL autocuidado de profesionales de la salud que atienden a víctimas de la violencia sexual. Rev Peru Ginecol Obstet 2006; 52:21-7.

44. Arjonilla S, Pelcastre B, Orozco E. Representación social del consumo del tabaco en una institución del salud. Salud Mental 2000; 23:2-12.

45. Añaños F. Representaciones sociales de los jóvenes sobre las drogas (alcohol, tabaco y cannabis) y su influencia en el consumo. Madrid: Dykinson S.L.; 2005.

46. Camarotti A, Kornblit A. Representaciones sociales y prácticas del consumo de éxtasis. Convergencia $2005 ; 12: 313-33$.

47. Alfonso A, Guerra G, Morejón Y. El cuidado y autocuidado de la voz en los maestros ambulantes y hospitalarios desde la concepción de promoción de salud. Revista Conrado 2015; 11:5-9.

48. Menéndez E, Di Pardo R. De algunos alcoholismos y algunos saberes. México DF: Centro de Investigaciones y Estudios Superiores en Antropología Social; 1996. 


\section{Abstract}

This study aimed to analyze the social representations of health self-care at work among urban bus drivers in Guadalajara, Mexico. The methodology was qualitative, with a multi-method design based on the theory of social representations. The first stage involved active observation sessions with the information recorded in field notes; in the second stage, the associative letter technique and successively hierarchized trees technique were applied to 20 drivers, submitting the data to a classical anal$y$ sis of the free associations; a third stage involved semi-structured interviews with 4 drivers, submitted to content analysis. The results indicate that structurally, the representation is centered on alimentation, presenting the concepts rest and corrective health care at peripheral levels. Procedurally, the represeentation centers in the psychosocial factors derived from the working conditions, while the rest of the work characteristics are seen as low risk to health. The self-care actions mentioned by the participants only focus on the workinging conditions perceived as risky. The social representation of health self-care at work is a collective mental construction, developed and modified through social interactions, which influences selfcare measures used against the risks perceived in the workplace.

Transportation; Occupational Health; Self Care

\section{Resumo}

O objetivo deste estudo foi analisar as representações sociais do autocuidado da saúde no trabalho dos motoristas de ônibus urbano de Guadalajara, México. A metodologia foi qualitativa, com uma abordagem multimétodo, baseada na teoria das representações sociais. Em uma primeira etapa foram efetuadas sessões de observação participante, a informação foi registrada em anotações de campo; na segunda fase foram aplicadas técnicas de cartas associativas e momentos hierárquicos sucessivos a 20 motoristas, cujos dados foram submetidos à análise clássico das associações livres; em uma terceira etapa foram realizadas entrevistas semiestruturadas a quatro motoristas, sendo submetidas a análises de conteúdo. Os resultados indicam que, estruturalmente, a representação está focada na alimentação, apresentando-se em níveis periféricos o descanso, junto com o cuidado correto da saúde. Processualmente, a representação vira em torno dos fatores psicossociais, que se desprendem das condições de trabalho, o resto das características da atividade laboral foi considerado pouco arriscado para a saúde. As ações de autocuidado citadas foram focadas às condições de trabalho percebidas como arriscadas. A representação social do autocuidado da saúde no trabalho é uma construção mental coletiva, desenvolvida e modificada através da interação social, a qual influencia as ações de autocuidado empregadas frente aos riscos existentes no entorno laboral.

Transportes; Saúde do Trabalhador; Autocuidado

Recibido el 27/Ago/2015

Versión final presentada el 23/May/2016

Aprobado el 02/Jun/2016 